\title{
The dopamine D2 agonist quinpirole disrupts attention to temporal signals without selectively altering the speed of the internal clock
}

\author{
LIANNE STANFORD and ANGELO SANTI \\ Wilfrid Laurier University, Waterloo, Ontario, Canada
}

\begin{abstract}
Three groups of rats were trained to discriminate between $2 \mathrm{sec}$ and $8 \mathrm{sec}$ of darkness by responding to either the left or the right lever. Following acquisition of this temporal discrimination, psychophysical functions were obtained by presenting unreinforced signals of intermediate duration. Two groups of rats were trained with saline and subsequently tested with the specific D2 dopamine agonist quinpirole $(0.08 \mathrm{mg} / \mathrm{kg})$. One of these groups was naive to the drug prior to testing (DN), whereas the other had exposure to the drug but not during training sessions (DE). A third group (DT) was trained under quinpirole and tested with saline. The temporal discrimination was acquired rapidly and equivalently in Groups DN and DE. However, rats in Group DT were severely impaired in acquiring the discrimination. During psychophysical testing, quinpirole disrupted the accuracy of temporal discrimination equivalently in Groups DN and DE. Both the Weber fraction (WF) and the difference limen (DL) increased significantly in Groups DN and DE; however, the point of subjective equality (PSE) was not affected. In Group DT, the shift to saline during psychophysical testing did not result in any changes to the PSE, DL, or WF. These findings are not consistent with the hypothesis that the speed of the internal clock is selectively affected by D2 dopaminergic manipulations. Prior exposure to the drug does not appear to be a critical variable in the failure to observe a selective adjustment of the internal clock. The D2 agonist quinpirole appears to affect the accuracy of temporal discriminations generally, without altering the speed of the internal clock.
\end{abstract}

Much of the research on animals' ability to perceive and remember time has benefited from the theoretical framework provided by an information-processing model of temporal processing. Church (1984) proposed an internal clock model that depicts how an animal perceives and remembers the duration of an event and decides if it is comparable to other reinforced durations. Church's model is composed of two stages: the clock stage--consisting of the pacemaker, switch, and accumulator - and the memory stage, which includes both reference and working memory. Closure of the switch allows pulses from the pacemaker to travel to an accumulator. The accumulated pulses on each trial are compared with previously reinforced durations stored in reference memory, and a response decision is made.

The point of subjective equality (PSE), or that point at which an animal chooses the response alternative reinforced following the long duration $50 \%$ of the time, has

This research was supported by Grant OGPOOD6378 from the Natural Sciences and Engineering Research Council of Canada to A.S. The article is based on a master's thesis submitted by the first author to Wilfrid Laurier University. The authors thank Linda Parker and Keith Horton for their comments at various stages of this research and Robert $\mathrm{V}$. MacDonald for help in collecting some of the data. L.S. is now in the Psychology and Neuroscience Program at Dalhousie University. Correspondence should be addressed to A. Santi, Department of Psychology, Wilfrid Laurier University, Waterloo, ON N2L 3C5, Canada (email: asanti@mach1.wlu.ca). been used to study animals' perception of event duration. Maricq, Roberts, and Church (1981) trained rats to choose between a left and a right lever following blackouts of different durations. Seven signal durations were presented, but only the two extreme signal durations were rewarded after a correct choice. On saline test sessions, the PSE was close to the geometric mean between the two extreme signal durations. Methamphetamine shifted the PSE to the left by approximately $10 \%$, relative to the PSE on saline control sessions. Maricq et al. reasoned that this was demonstrative of a change in clock speed, because the change in the PSE was positively related to the signal range being timed. Maricq and Church (1983) replicated the finding of a leftward shift in the PSE by methamphetamine. In addition, they demonstrated that haloperidol, a dopamine-receptor blocker, shifted the PSE rightward, whereas a combination of methamphetamine and haloperidol produced the same PSE as that found on saline control sessions.

Using similar procedures, Meck (1983) provided further support for the hypothesis that dopamine affects the clock stage of the internal clock. He reasoned that, if the clock stage was affected by a drug, the effects of that drug would be attenuated with repeated exposure, since the rats would learn to rescale the durations. In addition, abrupt termination of exposure to the drug would result in a shift of the psychophysical function in a direction opposite to that originally produced by the drug. However, if memory was affected, the shift in the psychophysical function 
would be permanent, and termination of chronic exposure would not result in a rebound effect. Meck (1983) found that methamphetamine shifted the PSE to the left by about $10 \%$. When methamphetamine administration was terminated, the PSE was shifted to the right. Haloperidol produced complementary effects to methamphetamine but in the opposite direction. Meck (1983) concluded that the effective level of dopamine determines the setting of clock speed in the rat, with methamphetamine increasing clock speed and haloperidol decreasing it.

A further advance in pharmacologically isolating the internal clock derives from a study by Meck (1986), in which he related the binding affinity of various dopamine antagonists to their potency in producing a temporary rightward shift in psychophysical timing functions. There are at least three different dopamine receptor subtypes: D1 (adenylate cyclase-linked), D2 (nonadenylate cyclaselinked), and D3 (presynaptic). Meck (1986) discovered that affinity for the dopamine D2 receptor best predicted the efficacy of dopamine antagonists in decreasing clock speed, and he concluded that dopamine D2 receptors played a major role in controlling the clock component of the internal clock.

Although this research has presented a compelling analysis of the role of dopamine in time perception, several studies in both rats (Lejeune et al., 1995; Rapp \& Robbins, 1976; Santi, Weise, \& Kuiper, 1995) and pigeons (Santi et al., 1995; Stubbs \& Thomas, 1974) have reported effects of dopaminergic manipulations that are not consistent with this analysis. Rapp and Robbins trained rats to make a left-lever response following a 3-sec tone and a right response following a $7-\mathrm{sec}$ tone. At the highest dose $(0.8 \mathrm{mg} / \mathrm{kg})$ of $d$-amphetamine administered, accuracy was disrupted, and 4 out of 5 rats exhibited a bias toward short responses. D. A. Stubbs and Thomas trained pigeons to peck a red comparison stimulus following shortduration samples and a green comparison stimulus following long-duration samples. They found that amphetamine had greater effects on longer sample durations, regardless of the absolute lengths of those durations. That is, amphetamine produced a choose-short response bias like that produced by increasing the length of delay intervals (Spetch, 1987). Lejeune et al. failed to observe a choose-long bias in time estimation after the very first injection of amineptine (a drug believed to induce dopamine release and to block dopamine uptake). They concluded that drugs modulating the dopaminergic system do not selectively target time mechanisms specifically. Santi et al. found that, for both rats and pigeons, amphetamine reduced the overall accuracy of temporal discriminations without producing any evidence of a bias to overestimate time. These results indicate that amphetamine-induced impairment of temporal discrimination performance involves more than simple direct influences on internal clock processes.

To summarize, research by Meck and his colleagues has provided considerable support for the involvement of dopamine in altering the speed of the internal clock
(Maricq \& Church, 1983; Maricq et al., 1981; Meck, 1983 , 1986; Meck \& Church, 1983). However, other studies of dopaminergic effects on temporal discriminations in both rats and pigeons have failed to produce a chooselong response bias that would be consistent with a speeding of the internal clock (Lejeune et al., 1995; Rapp \& Robbins, 1976; Santi et al., 1995; D. A. Stubbs \& Thomas, 1974). It is widely recognized that dopaminergic agents may not exclusively affect processes related to time discrimination. For example, there is considerable evidence that dopaminergic drugs such as amphetamine affect nontemporal working and reference memory (Bushnell \& Levin, 1993; Packard \& White, 1989). Nevertheless, it is important to know precisely what experimental conditions are necessary in order to produce selective clock effects such as those reported by Meck (1996). Drug dosage and type of drug can probably be ruled out, because these parameters were the same in Meck (1983) and in Santi et al. Three other experimental conditions that might be relevant are the psychophysical method itself, prior experience with the drug, and the method of data analysis.

Of those studies producing inconsistent results, only Stubbs and Thomas (1974) obtained psychophysical functions, but the psychophysical method that they used differed in several ways from the standard bisection procedure used by Meck (see Fetterman, 1995). Second, in all of the studies producing inconsistent results, the animals had no prior experience with the drug. In Meck (1983), a specialized procedure was used. Rats were trained to discriminate temporal durations under saline, and they received a drug, such as amphetamine, outside the training paradigm on nontraining days. Consequently, although these animals were experienced with the effects of the drug, they had never experienced these effects while performing the temporal discrimination task until the first test session. It could be argued that the initial exposure to a drug such as amphetamine has a general disruptive effect on behavior that prevents selective clock effects from being detected. Maricq et al. (1981) noted that, "in order to determine if clock rate is affected, it is essential to observe the effect of a drug that does not lead to a marked attenuation of temporal control" (p. 29). Ensuring that animals are familiar with the drug prior to assessing its effects on time perception may be necessary to observe selective clock effects.

Finally, the method of data analysis may be critical for demonstrating a speeding of the clock under amphetamine. In studies by Maricq et al. (1981), Maricq and Church (1983), and Meck (1983), the psychophysical functions were calculated on the basis of responses faster than the mean latency of responses with a correction for responses not controlled by time. Other studies of amphetamine's effects on temporal discriminations in both rats (Lejeune et al., 1995; Rapp \& Robbins, 1976; Santi et al., 1995) and pigeons (Santi et al., 1995; Stubbs \& Thomas, 1974) have included the responses of all trials in their analyses.

The present study was designed to extend current knowledge of the effects of dopaminergic manipulations 
on time perception, with respect to both pharmacology and behavioral testing procedures. With respect to pharmacology, this study examined the effects of a specific $D 2$ receptor agonist, quinpirole, on time perception. The effects of specific D2 receptor agonists on psychophysical timing functions have not previously been reported for rats. With respect to behavioral testing, this study allowed assessment of how important the three variables of prior drug exposure, psychophysical testing method, and method of data analysis are to the detection of selective clock effects. The role of prior drug exposure was assessed by manipulating the training and testing conditions for three groups of rats. Two groups of rats were trained with saline and subsequently tested with the specific D2 dopamine agonist quinpirole. One of these groups was naive to the drug prior to testing (DN), whereas the other had prior exposure to the drug but not during training sessions (DE). A third group (DT) was trained under quinpirole and tested with saline. According to the biological articulation of the internal clock model (Meck, 1996), the group trained under quinpirole (DT) should show an immediate rightward shift in the psychophysical function (increase in the PSE) when tested under saline. The group trained under saline but experienced with quinpirole (DE) should show an immediate leftward shift in the psychophysical function (decrease in the PSE) when tested with quinpirole. Finally, if the effect of drug novelty was responsible for discrepancies in the research literature noted earlier, then animals with no prior drug experience (DN) would be generally disrupted, as indicated by a loss of accuracy at the endpoints, and no internal clock manipulation would be found. The psychophysical testing method was the same as that used by Meck (1983). Finally, the data were analyzed in two ways - both by including all trials during test sessions and by eliminating trials in which the latency of responding was greater than $3.0 \mathrm{sec}$ (see Meck, 1986).

\section{METHOD}

\section{Subjects}

Twenty-four adult male Sprague-Dawley rats (Charles River, Canada), naive in drug and temporal discrimination experiments, started as subjects. They were individually housed in clear Plexiglas shoebox cages in a vivarium with 12:12-h light:dark cycles, with fluorescent lights on at 8:00 a.m. and ad-lib access to water. During testing, they were food deprived and maintained at approximately $85 \%$ of their normal body weight, with supplemental feeding of LabDiet for rodents (PMI Feeds). At the beginning of the experiment, the rats were approximately 90 days old.

\footnotetext{
Apparatus

Four Coulbourn operant chambers (Model E10-10), individually housed in isolation chambers (Model E10-20) and equipped with baffled exhaust fans, were used. On the front wall of the chamber, two retractable levers (Model E23-07) in two of the boxes and Model E23-17 in the other two were positioned on either side of a pellet feeder (Model E14-06) approximately $3 \mathrm{~cm}$ from the grid floor and $14 \mathrm{~cm}$ apart. The pellet feeder, placed in the center of the front wall, with the opening approximately $3 \mathrm{~cm}$ from the floor of the chamber, provided access to $45-\mathrm{mg}$ pellets (Bioserve Universal
}

Research Test Diets grain-based rodent pellets). A houselight (Model E1 1-01, bulb SL 1819x), positioned $6.5 \mathrm{~cm}$ directly above the pellet feeder and reflecting toward the ceiling of the chamber, remained on throughout each trial. Offset of the houselight was the carrier of the temporal signal. All events and nses were arranged and recorded by a microcomputer system luuded in the same room.

\section{Drug}

The highly effective D2 agonist quinpirole was used (Research Biochemicals International, Natick, MA). The dose of $0.08 \mathrm{mg} / \mathrm{kg}$ for quinpirole was administered interperitonally using a distilled water vehicle and dissolved so that injection volumes were $1 \mathrm{ml} / \mathrm{kg}$. This dose was used for all rats during the first 45 sessions. At this point, the DN and DE groups had completed the study, and the dose of quinpirole was reduced to $0.04 \mathrm{mg} / \mathrm{kg}$ for the DT group in order to facilitate their acquisition of the two-signal training task. Control injections utilized physiological saline $(1 \mathrm{ml} / \mathrm{kg})$.

The $0.08-\mathrm{mg} / \mathrm{kg}$ dosage of quinpirole was selected on the basis of previous research conducted by Bushnell and Levin (1993). They found that a $0.056-\mathrm{mg} / \mathrm{kg}$ dose of quinpirole and a $1.00-\mathrm{mg} / \mathrm{kg}$ dose of $d$-amphetamine produced a similar disruption of spatial delayed nonmatching-to-position in rats. In previous studies of timing in rats, a $1.5-\mathrm{mg} / \mathrm{kg}$ dose of methamphetamine has been used (Maricq et al., 1981; Meck, 1983; Meck \& Church, 1983; Santi et al., 1995). Consequently, the $0.056-\mathrm{mg} / \mathrm{kg}$ dose of quinpirole used in the Bushnell and Levin (1993) study was increased by approximately $50 \%$ in order to approximate the behavioral effects of a $1.5-\mathrm{mg} / \mathrm{kg}$ dose of amphetamine.

\section{Procedure}

The twenty-four rats were randomly assigned to one of three groups, so that each group consisted of 8 animals. One group of animals had quinpirole injections during training (drug trained, DT), and another group experienced quinpirole outside of the training paradigm (DE). The third group had no experience with quinpirole drug prior to testing (DN). Rats were trained 7 days per week between 0800 and $1400 \mathrm{~h}$.

Training and testing of the animals consisted of four stages: pretraining, two-signal training, seven-signal training, and seven-signal testing. During pretraining, several sessions of combined magazine and lever training were given. The rats were placed in the chamber with both the left and right levers retracted. Each trial commenced with the entry of the left or right lever into the chamber. The lever remained extended until it was pressed or $60 \mathrm{sec}$ had elapsed, whichever occurred first. Either event resulted in delivery of a food pellet and retraction of the lever. Pellet delivery produced an audible click, and the light in the magazine was illuminated for $0.5 \mathrm{sec}$. The houselight illuminated the chamber throughout each session. After four 60 -min sessions, those rats that had not adequately acquired the barpressing response were manually shaped to leverpress. Once leverpressing was established, two-signal discrimination training began.

Quinpirole and/or saline injections began during two-signal training and were maintained throughout the remainder of the experiment. There were two types of sessions: training/testing sessions and exposure sessions. During training/testing sessions, the rats were injected, placed in the operant chambers, and presented with the stimulus and response contingencies as described below. During exposure sessions, the animals were injected, placed in the operant test chambers with the levers retracted for $20 \mathrm{~min}$, but not exposed to any stimulus or response contingencies. The order of these two session types was randomized, with a maximum of 2 consecutive days of one type of session. The $\mathrm{DN}$ group received saline injections $30 \mathrm{~min}$ prior to the training/testing sessions, as well as prior to the exposure sessions. The DE group received saline injections $30 \mathrm{~min}$ prior to training/testing sessions and quinpirole $30 \mathrm{~min}$ prior to exposure sessions. The DT group received quinpirole injections $30 \mathrm{~min}$ 
prior to training/testing sessions and saline $30 \mathrm{~min}$ prior to exposure sessions.

Each session of two-signal training consisted of 50 presentations of each signal duration, with a maximum session duration of $60 \mathrm{~min}$. Half of the rats were trained to press the left lever following 2-sec offset of the houselight (short response) and to press the right lever following 8-sec offset of the houselight (long response). The other half of the rats were trained with the opposite contingencies. The right and left levers were counterbalanced for temporal durations across and within groups. A correct response was reinforced with a food pellet. An incorrect response initiated a correction procedure. The correction procedure consisted of a 5 -sec delay prior to a re-presentation of the same signal duration. A correct response during a correction trial resulted in delivery of a food pellet, presentation of an intertrial interval (ITI), and progression to the next trial. The ITI varied in duration $(5,10,15,20$, or $25 \mathrm{sec})$ within sessions. Only the first response choice on each trial was included in the recorded data.

Two-signal training continued until the average accuracy for short and long signals was consistently above $80 \%$ correct for each group. When the discrimination had been acquired, the probability of being rewarded for a correct response was reduced to .5. Intermittent reinforcement sessions continued until performance stabilized, and then seven-signal training began. Two rats in Group DT, which received quinpirole during training sessions, were not able to meet the $80 \%$ accuracy criterion after 84 training sessions and were dropped from the study.

Seven-signal training maintained the same conditions as two-signal training sessions, except that (1) each of the two extreme signal durations ( 2 and $8 \mathrm{sec}$ ) was presented with a probability of .25 on each trial and (2) on the remaining trials, one of five intermediate signal durations was presented, each with equal probability $(.10)$. The intermediate signal durations, as in Meck (1983), were spaced at approximately equal logarithmic intervals between the two signal durations used in training $(2.6,3.2,4.0,5.0$, and $6.4 \mathrm{sec})$. The rats were still rewarded with a probability of .5 for responding correctly to the 2- and the 8-sec signal, but responses to either lever following intermediate signals never resulted in food. During the eight sessions of seven-signal training, the drug injections administered were the same as those administered during the previous twosignal training sessions.

Seven-signal testing sessions occurred immediately after the eight sessions of seven-signal training. The conditions of sevensignal training were maintained, except that the drug injection conditions were reversed. The DT group now received saline injections prior to seven-signal test sessions and quinpirole prior to exposure sessions. The two groups that had no experience with quinpirole during training sessions (DN and $D E$ ) now received quinpirole injections prior to test sessions. Eight sessions of seven-signal testing were given.

\section{RESULTS}

\section{Acquisition}

Figure 1 shows the mean percent correct during twosignal training sessions for Groups DN, DE, and DT. Both the DN and the DE groups achieved the accuracy criterion within 23 sessions of training. Exposure to quinpirole on nontraining days in Group DE had no effect on the speed with which they acquired the temporal discrimination or the final level of accuracy achieved. Their acquisition functions were the same as Group DN, which had no exposure to quinpirole during two-signal training. However, exposure to quinpirole during training sessions themselves in Group DT had a substantial effect on the speed with which the discrimination was acquired. Ti: 2 data for Group DT only shows the data for the 6 rats that eventually acquired the discrimination. Overall accuracy in Group DT remained below $75 \%$ correct, even after 45 sessions of training. The dose of quinpirole was reduced to $0.04 \mathrm{mg} / \mathrm{kg}$ on the $46 \mathrm{th}$ session of training for Group DT, and accuracy improved on subsequent sessions. It is unclear whether the rats in this group would have eventually achieved an accuracy level of $80 \%$ without the reduction in drug dosage.

\section{Seven-Signal Training and Testing for Groups DN and DE}

The mean proportion of long responses are shown in Figure 2 as a function of signal duration during the eight sessions of seven-signal training and testing for Groups DN and DE. An analysis of variance (ANOVA) of these data, with group as a between-subjects factor and signal duration and phase (training vs. testing) as within-subjects factors, was conducted. There was no significant effect of group or phase $(F \mathbf{S}<1)$, but there was a main effect for signal duration $[F(6,84)=331.85, p<.001]$. The threeway interaction of group $\times$ phase $\times$ signal duration was not statistically significant $(F<1)$. However, the interactions of group $\times$ signal duration $[F(6,84)=2.77, p<$ $.05]$ and phase $\times$ signal duration $[F(6,84)=24.69, p<$ $.001]$ were significant. These two interactions are plotted in Figure 3. The group $\times$ signal duration interaction occurred because Group DE showed a sharper temporal discrimination function than did Group DN. This was particularly evident at the shorter signal durations of 2.0 and $2.6 \mathrm{sec}$, where Group DE made significantly fewer long responses than did Group DN $[F(1,14)=5.03$ and 5.88, $p \mathrm{~s}<.05]$. The phase $\times$ signal duration interaction was due to a flattening of the psychophysical function during quinpirole test sessions. At signal durations less than $4 \mathrm{sec}$, more long responses occurred during quinpirole test sessions than during saline training sessions. At signal durations of $4 \mathrm{sec}$ or more, fewer long responses occurred during quinpirole test sessions than during saline training sessions. The differences in the proportion of long responses between the saline training phase and the quinpirole test phase were statistically significant at each signal duration $[F \mathrm{~s}(1,14)=37.09,28.23,16.77,5.11$, $10.45,10.89$, and $28.47, p$ s from $<.05$ to $<.001$ ]

PSEs were estimated from the psychophysical functions for each rat by conducting linear regressions of the proportion of long responses for each of three adjacent signal durations. The regression equation with the greatest slope for each rat was used to estimate the PSE by calculating the signal duration associated with $50 \%$ of the long responses. For Group DN, the mean PSE was $3.91 \mathrm{sec}$ under saline and $3.97 \mathrm{sec}$ under quinpirole. For Group DE, the mean PSE was $3.86 \mathrm{sec}$ under saline and $4.21 \mathrm{sec}$ under quinpirole. An ANOVA of the PSE data failed to reveal any significant main effects or interactions. The 


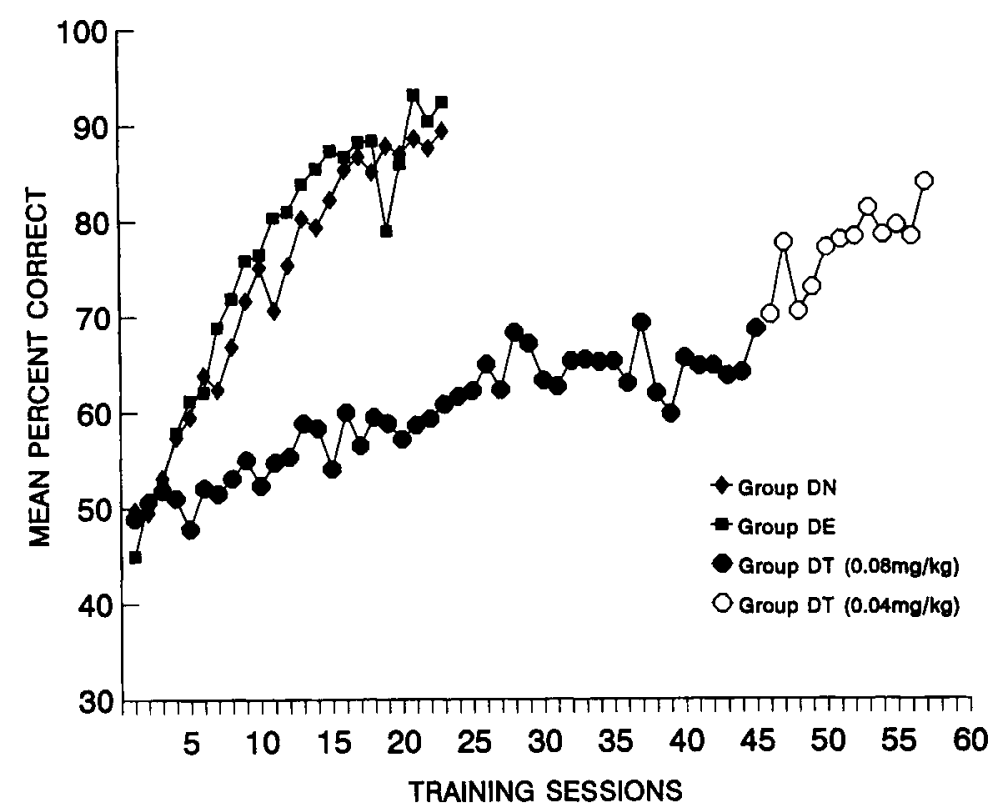

Figure 1. Mean percent correct as a function of sessions during two-signal training for the three experimental groups. The group (DN), trained under saline (without exposure to quinpirole), is indicated by the diamonds. The group (DE), trained under saline (with exposure to quinpirole), is indicated by the squares. The group (DT), trained under quinpirole, is indicated by the closed circles for sessions with the $0.08-\mathrm{mg} / \mathrm{kg}$ dosage and by the open circles for sessions with the $0.04-\mathrm{mg} / \mathrm{kg}$ dosage.

overall mean PSE under saline was $3.89 \mathrm{sec}$, whereas under quinpirole it was $4.09 \mathrm{sec}$. These values are near the geometric mean of the physical times of the two reinforced signals.

The regression equations were also used to calculate difference limens (DL), which represent the average difference between the signal duration associated with $75 \%$ long responses and the signal duration associated with $25 \%$ long responses. The mean DL was $1.34 \mathrm{sec}$ for Group $\mathrm{DN}$ and $0.95 \mathrm{sec}$ for Group DE. During training under saline, the mean DL was $0.79 \mathrm{sec}$, whereas during testing under quinpirole, it was $1.49 \mathrm{sec}$. An ANOVA indicated a significant main effect of group and phase $[F(1,14)=$ 4.83 and $16.19, p<.05$ and .001$]$. Consequently, Group DE displayed greater sensitivity to time than did Group $\mathrm{DN}$, and the rats in both groups were more sensitive to time when tested under saline than they were under quinpirole. The group $\times$ phase interaction was not statistically significant.

The Weber fraction (WF) was calculated as DL/PSE. The mean WFs were 0.34 for Group DN and 0.23 for Group DE. During training under saline, the mean WF was 0.20 , whereas, during testing under quinpirole, it was 0.37 . An ANOVA of the WFs produced similar results to those obtained for the DLs. Both main effects were significant $[F(1,14)=11.28$ and $6.07, p s<.001$ and .05$]$, but the interaction was not. These analyses indicate that quinpirole affected the quality of the temporal discrimi- nation (DLs and WFs) without selectively increasing the speed of the internal clock (i.e., lowering the PSE).

\section{Seven-Signal Training and Testing for Group DT}

The mean proportion of long responses as a function of signal duration during seven-signal training under quinpirole and testing under saline are shown in Figure 4. Although the main effect of signal duration was statistically significant $[F(6,30)=42.11, p<.001]$, neither the phase nor the phase $\times$ signal duration interaction was statistically significant. In addition, there was no significant difference between training and testing for the PSE, DL, or WF. The mean values for the PSE, the DL, and the WF was $4.07 \mathrm{sec}, 1.44 \mathrm{sec}$, and 0.36 , respectively. These values of the PSE, DL, and WF are very similar to those obtained during quinpirole test sessions in Group DN and Group DE (4.21 sec, $1.49 \mathrm{sec}$, and 0.37, respectively).

\section{Additional Analyses}

An additional set of data analyses were conducted, using procedures similar to those reported by Meck (1986). Choice responses with latencies greater than $3 \mathrm{sec}$ were not included, on the assumption that they are not well controlled by time. During the saline training phase, an average $2.7 \%$ of the data for Group DN and $0.3 \%$ of the data for Group DE were discarded by the 3-sec latency cutoff. During the quinpirole test phase, an average of $13.8 \%$ of the data for Group DN and $8.6 \%$ of the data 


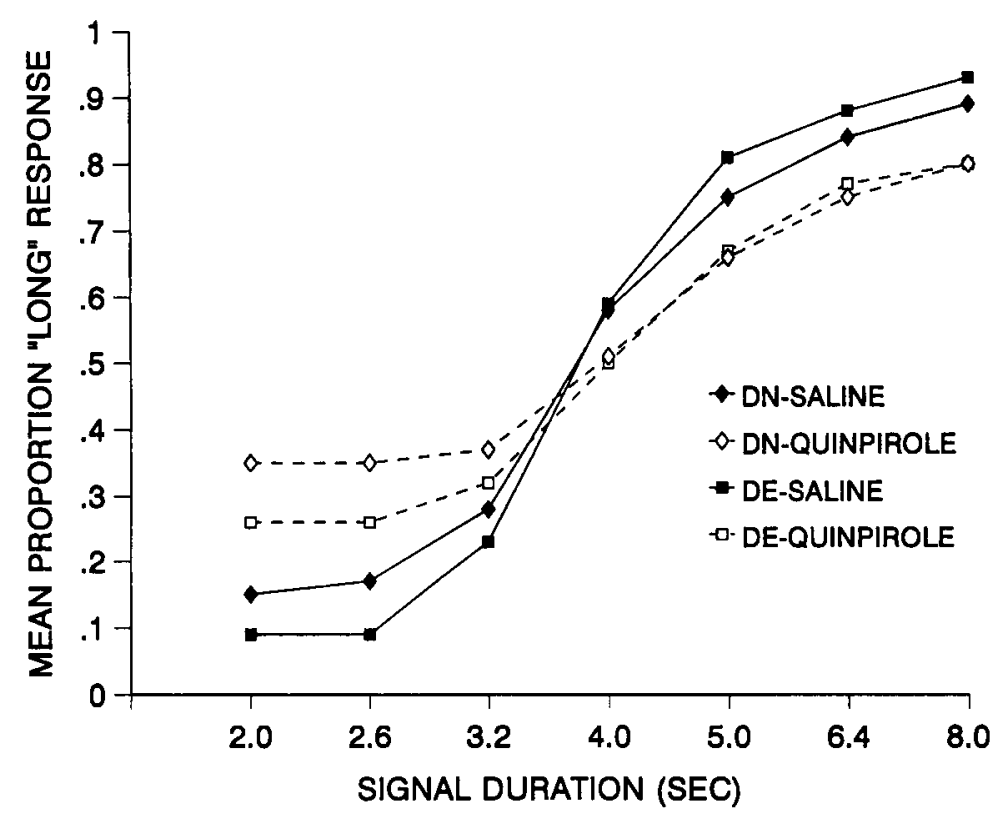

Figure 2. The mean proportion of long responses as a function of signal duration during the eight sessions of seven-signal training and testing for Group DN and Group DE.

for Group DE were discarded. The percentage of trials excluded was significantly greater under quinpirole than under saline $[F(1,14)=31.49, p<.001]$. All of the analyses conducted on trials that met the latency criterion were consistent with the main findings described earlier, with two exceptions. The difference between Group DN and Group DE at signal durations of 2.0 and $2.6 \mathrm{sec}$ was no longer significant. In addition, there was a statistically significant increase in the PSE between saline training sessions and quinpirole testing sessions in these two groups [3.9 vs. $4.2 \mathrm{sec} ; F(1,14)=4.79, p<.05$ ]. This increase in the PSE would be more consistent with quinpirole's decreasing, rather than increasing, clock speed. Most importantly, however, even when only those trials that met the latency criterion were used, there was a significant increase in both the DL [0.78 vs. $1.35 \mathrm{sec}$; $F(1,14)=14.95, p<.001]$ and the WF $[0.19$ vs. 0.32 ; $F(1,14)=11.87, p<.001]$ when the psychophysical functions were obtained under quinpirole rather than under saline.

For Group DT, an average of $5.8 \%$ of the trials were discarded by the latency criterion. However, the results of the analyses conducted on these data were the same as those reported without discarding any trials.

\section{DISCUSSION}

Quinpirole disrupted acquisition of the temporal discrimination when it was administered during training sessions (Group DT) but had no effect on acquisition when it was administered outside of the training sessions (Group DE vs. Group DN). Meck (1983) also found that the rate of acquisition of temporal discriminations was lowered for those rats trained under the dopamine agonist methamphetamine. Exactly how quinpirole or other dopamine agonists disrupt learning is uncertain. Quinpirole has a variety of effects in different species and experimental preparations, including affecting processes such as memory (Arnsten, Cai, Steere, \& Goldman-Rakic, 1995; Gasbarri et al., 1997; Packard \& McGaugh, 1994), the rewarding effect of brain stimulation (Nakajima \& Patterson, 1997; Ranaldi \& Beninger, 1994), and behavioral sensitization (Szechtman, Talangbayan, \& Eilam, 1993).

The psychophysical functions obtained under saline training conditions in Groups DN and DE had properties that were similar to those previously reported (Church \& Deluty, 1977; Maricq \& Church, 1983; Maricq et al., 1981; Meck, 1983; Stubbs, 1968). The PSE was approximately at the geometric mean of the two reinforced durations. In addition, the specific values for the PSE, DL, and WF in this study (3.89 sec, $0.79 \mathrm{sec}$, and 0.21 , respectively) compare very favorably with those reported by Meck (1983) (4.03 sec, $0.85 \mathrm{sec}$, and 0.21, respectively). However, when Groups DN and DE were tested under quinpirole, there was no detectable horizontal leftward shift in the psychophysical function, which would be expected if the speed of a rat's internal clock had been selectively increased by quinpirole. Instead, the psychophysical functions tended to flatten under quinpirole, indicating a loss in the ability to discriminate time that could reflect a disruption of attention to temporal signals. This reduction of temporal discrimination accuracy was not simply the result of drug novelty, because the rats that had experienced quinpirole prior to testing (Group 

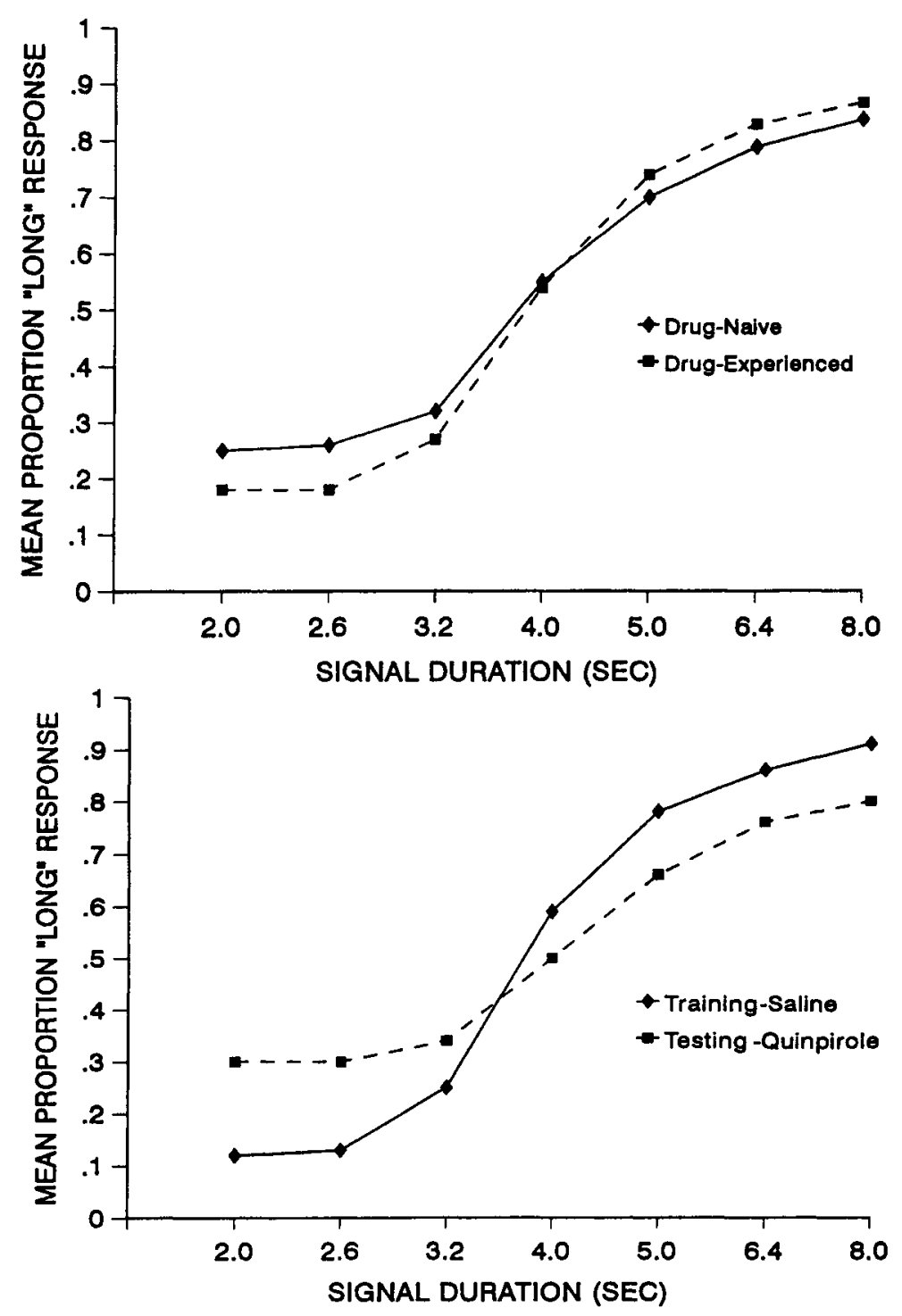

Figure 3. The interaction of group $\times$ signal duration (top) and study phase $\times$ signal duration (bottom) obtained in Group DN and Group DE during training with saline and testing with quinpirole.

DE) were as disrupted as those rats that had no prior drug experience (Group DN). Thus, the novelty of quinpirole is not an important factor in determining whether it produces a flattening, as opposed to a horizontal, shift in psychophysical time functions.

Just as administration of quinpirole failed to produce a significant leftward shift in the psychophysical time functions for Groups DN and DE, the shift from quinpirole to saline injections failed to produce a significant rightward shift in the function for Group DT. The shift from quinpirole to saline had no significant effect on the psychophysical time function at all. In fact, the PSE, DL, and WF in Group DT were very similar to those obtained during quinpirole test sessions in Group DN and Group $D E$. This indicates that the detrimental effect of quinpi- role on the perception of time was not attenuated with repeated exposure to the drug.

Although some previous research has provided considerable support for the involvement of dopamine in the modification of the speed of the internal clock (Maricq et al., 1981; Meck, 1983, 1986; Meck \& Church, 1983), other studies in both rats and pigeons have failed to produce results consistent with a speeding of the internal clock (Lejeune et al., 1995; Rapp \& Robbins, 1976; Santi et al., 1995; Stubbs \& Thomas, 1974). Neither procedural details nor methods of analysis provide a basis for distinguishing between studies that show effects on clock rate and those that do not. Drug dosage and type of drug can be ruled out, because these parameters were identical in Meck (1983) and Santi et al. Previous exposure to 


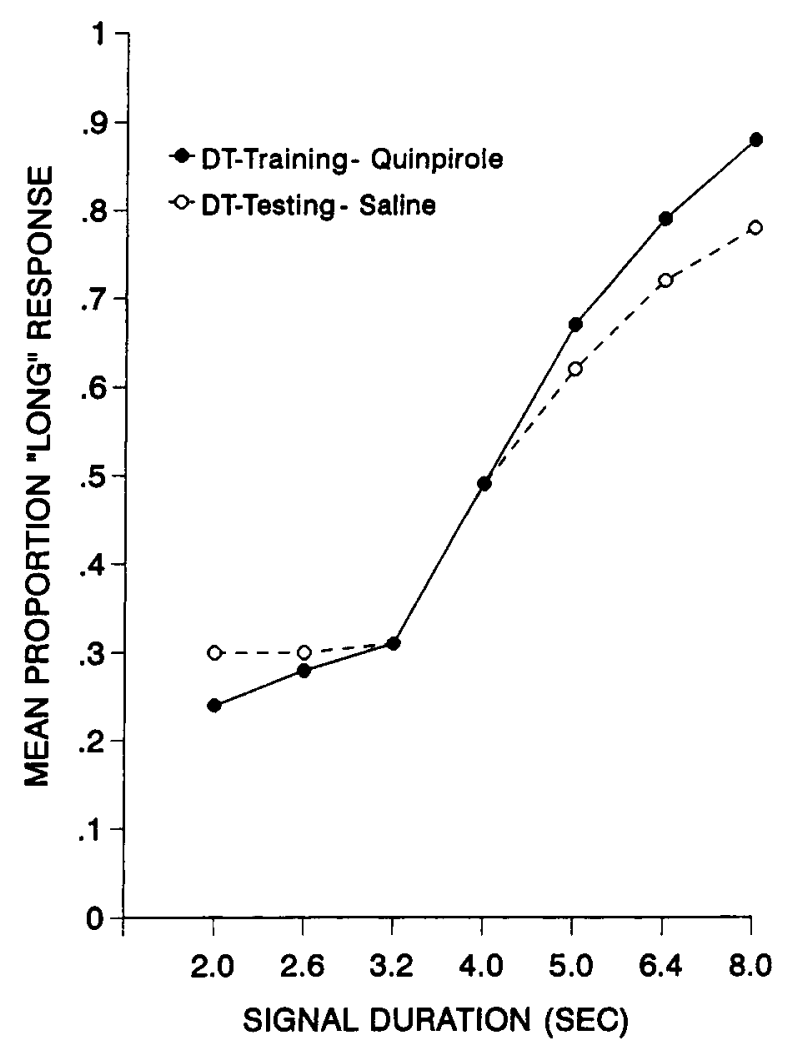

Figure 4. The mean proportion of long responses as a function of signal duration during seven-signal training under quinpirole and testing under saline for Group DT.

the drug does not seem to be important, for two reasons. First, in the present study, prior exposure to the drug was manipulated, and no major differences in quinpirole's effect on psychophysical time functions were observed between Group DN and Group DE. Second, Meck and Church were able to obtain selective clock effects and no attenuation of temporal control in rats that had no prior exposure to methamphetamine. The shift in the psychophysical functions that occurred in Meck and Church was identical to that which had occurred in a previous study (Meck, 1983) in which rats had been given prior exposure to methamphetamine.

The use of a psychophysical testing procedure to assess the effects of dopaminergic manipulations also does not appear to be a significant factor. Although the current psychophysical method was the same as that used by Meck (1983) and it produced values for the PSE, DL, and WF under saline control conditions that were very similar to those previously reported, there was no evidence for a dopamine-induced speeding of the internal clock.

Finally, in the present study, analyses were also conducted after eliminating trials on which the response latency was greater than $3.0 \mathrm{sec}$. These analyses were consistent with those conducted on the entire data set in showing that there was an attenuation of temporal control but no evidence of a speeding of the internal clock. On the basis of the present study and previously published research, it appears as if neither the type of drug, the drug dosage, prior exposure to the drug, the use of a psychophysical testing procedure, nor the use of a response latency criterion can easily account for the presence or absence of selective clock effects following dopaminergic manipulations.

Two main types of behavioral procedures have been used in the study of drug effects and timing in animals: the temporal bisection procedure and the peak-interval procedure. The temporal bisection procedure was used in the present study, and our discussion has focused primarily on research that also used this procedure. In the peakinterval procedure, animals are trained on a fixed-interval schedule to respond for food after a specified signaled interval has elapsed. On test trials, the duration of the signal is extended, and no food is delivered. Research examining the effects of amphetamine (Kraemer, Randall, Dose, \& Brown, 1997) as well as specific D1 and D2 agonists (Frederick \& Allen, 1996) has been successful in replicating the decrease in peak time reported by Maricq et al. (1981). However, Frederick and Allen (1996) were unable to demonstrate that affinity for the D2 receptor was related to the decrease in peak time, nor were they able to obtain a significant increase in peak time when D 1 and D2 antagonists were administered. Because drugs might affect processes involved in the behavioral expression of timing rather than timing itself, the temporal bisection procedure is methodologically preferred over the peakinterval procedure. However, additional research with both procedures is needed in order to confidently determine whether the dopamine system is directly involved in timing processes.

There are limitations to the interpretation of the present results that require some comment. First, the conclusions are based on the use of a single dose of quinpirole $(0.08 \mathrm{mk} / \mathrm{kg})$. Although this dose appears to alter the accuracy of timing generally, without selectively altering the speed of the internal clock, it is possible that different doses of quinpirole might alter clock speed. If such a dose could be found, it would be worthwhile to train animals with different training durations in order to determine whether a proportional shift in the timing function occurs. Second, the inability of quinpirole to produce a selective clock effect does not rule out an effect of dopamine on clock speed. Although clock speed can be decreased by selective D2 antagonists, it may be that clock speed can only be increased by drugs, such as amphetamine, that activate both $\mathrm{D} 1$ and $\mathrm{D} 2$ receptors. In addition, the clock rate-increasing effects of drugs such as amphetamine may be observed in some but not all of the procedures used to study temporal discriminations in animals.

In summary, the present findings are not consistent with the hypothesis that the speed of the internal clock is selectively affected by D2 dopaminergic agonists. Prior exposure to quinpirole does not appear to be a critical variable in the failure to observe a selective adjustment of the internal clock. Quinpirole appears to reduce attention to 
temporal signals and to reduce the accuracy of temporal discriminations without altering the speed of the internal clock.

\section{REFERENCES}

Arnsten, A. F. T., Cai, J. X., Steere, J. C., \& Goldman-Rakic, P. S (1995). Dopamine D2 receptor mechanisms contribute to age-related cognitive decline: The effects of quinpirole on memory and motor performance in monkeys. Journal of Neuroscience, 15, 3429-3439.

BushNELL, P. J., \& LEVIN, E. D. (1993). Effects of dopaminergic drugs on working and reference memory in rats. Pharmacology, Biochemistry \& Behavior, 45, 765-776.

ChurCh, R. M. (1984). Properties of the internal clock. In J. Gibbon \& L. Allen (Eds.), Timing and time perception (Annals of the New York Academy of Sciences, Vol. 423, pp. 566-582). New York: New York Academy of Sciences.

Church, R. M., \& Deluty, M. Z. (1977). Bisection of temporal intervals. Journal of Experimental Psychology: Animal Behavior Processes, 3, 216-228.

Fetrerman, J. G. (1995). The psychophysics of remembered duration. Animal Learning \& Behavior, 23, 49-62.

FREDERICK, D. L., \& AlLEN, J. D. (1996). Effects of selective dopamine D1 and D2 agonists and antagonists on timing performance in rats. Pharmacology, Biochemistry \& Behavior, 53, 759-764.

Gasbarri, A., Sulli, A., Pacitti, C., Puglisi-Allegra, S., Cabib, S., Castellano, C., Introini-Collison, I., \& McGaugh, J. L. (1997). Strain-dependent effects of D2 dopaminergic and muscariniccholinergic agonists and antagonists on memory consolidation processes in mice. Behavioral Brain Research, 86, 97-104.

Kraemer, P. J., Randall, C. K., Dose, J. M., \& Brown, R. W. (1997). Impact of $d$-amphetamine on temporal estimation in pigeons tested with a production procedure. Pharmacology, Biochemistry \& Behavior, 58, 323-327.

Lejeune, H., Hermans, I., Mocaer, E., Rettori, M., Poignant, J. C., \& Richelle, M. (1995). Amineptine, response timing, and time discrimination in the albino rat. Pharmacology, Biochemistry \& Behavior, 51, 165-173.

Marice, A. V., \& Church, R. M. (1983). The differential effects of haloperidol and methamphetamine on time estimation in the rat. Psychopharmacology, 79, 10-15.

MaricQ, A. V., Roberts, S., \& ChUrch, R. M. (1981). Methamphetamine and time estimation. Journal of Experimental Psychology: Animal Behavior Processes, 7, 18-30.
MEck, W. H. (1983). Selective adjustment of the speed of internal clock and memory processes. Journal of Experimental Psychology: Animal Behavior Processes, 9, 171-201.

MEcK, W. H. (1986). Affinity for the dopamine D2 receptor predicts neuroleptic potency in decreasing the speed of an internal clock. Pharmacology, Biochemistry \& Behavior, 25, 1185-1189.

MECK, W. H. (1996). Neuropharmacology of timing and time perception. Cognitive Brain Research, 3, 227-242.

Meck, W. H., \& ChURCH, R. M. (1983). A mode control model of counting and timing processes. Journal of Experimental Psychology: Animal Behavior Processes, 9, 320-334.

Nakajima, S., \& Patterson, R. L. (1997). The involvement of dopamine D2 receptors, but not D3 or D4 receptors, in the rewarding effect of brain stimulation in the rat. Brain Research, 760, 74-79.

PaCKarD, M. G., \& McGaugh, J. L. (1994). Quinpirole and $d$-amphetamine administration posttraining enhances memory on spatial and cued discriminations in a water maze. Psychobiology, 22, 54-60.

PACKARD, M. G., \& WhITE, N. M. (1989). Memory facilitation produced by dopamine agonists: Role of receptor subtype and mnemonic requirements. Pharmacology, Biochemistry \& Behavior, 33, 511-518.

RANALDI, R., \& BENINGER, R. J. (1994). The effects of systemic and intercerebral injections of D1 and D2 agonists on brain stimulation reward. Brain Research, 651, 283-292.

RAPP, D. L., \& RoBBinS, T. W. (1976). The effects of $d$-amphetamine on temporal discrimination in the rat. Psychopharmacology, 51, 91-100.

SANTI, A., WeISE, L., \& KuIPER, D. (1995). Amphetamine and memory for event duration in rats and pigeons: Disruption of attention to temporal samples rather than changes in the speed of the internal clock. Psychobiology, 23, 224-232.

SPETCH, M. L. (1987). Systematic errors in pigeons' memory for event duration: Interaction between training and test delay. Animal Learning \& Behavior, 15, 1-5.

STuBBS, [D.] A. (1968). The discrimination of stimulus duration by pigeons. Journal of the Experimental Analysis of Behavior, 11, 223-238.

Stubis, D. A., \& Thomas, J. R. (1974). Discrimination of stimulus duration and $d$-amphetamine in pigeons: A psychophysical analysis. Psychopharmacologia, 36, 313-322.

Szechtman, H., Talangbayan, H., \& Eilam, D. (1993). Environmental and behavioral components of sensitization induced by the dopamine agonist quinpirole. Behavioural Pharmacology, 4, 405-410.

(Manuscript received December 4, 1997; revision accepted for publication May 13, 1998.) 